Anaesthesist 2013 $\cdot 62: 252-253$

DOI 10.1007/s00101-013-2164-z

Online publiziert: 19. April 2013

(c) Springer-Verlag Berlin Heidelberg 2013

\title{
J. Biscoping
}

Klinik für Anaesthesie und Operative Intensivmedizin, St. Vincentius-Kliniken gAG, Karlsruhe

\section{TRALI - ein Lehrstück für Hämovigilanz}

gerer Zeit darum sehr verdient gemacht $[1,3,4]$. Einen Durchbruch für die klinische Akzeptanz bedeuteten jedoch die jährlichen Daten des „SHOT report“, die TRALI eine herausragende Bedeutung für schwerste und z. T. tödliche Transfusionszwischenfälle zuwiesen [5]. Diese beeindruckende Sammlung und Darstellung von ernsthaften Zwischenfällen in direktem Zusammenhang mit klinischer Hämotherapie in England war nur durch ein dort konsequent umgesetztes Meldesystem möglich, das in Bezug auf Hämovigilanz Vorbildcharakter für ganz Europa hat. Doch auch in Deutschland existieren mit dem Transfusionsgesetz (TFG) und dem Arzneimittelgesetz (AMG) verbindliche Regelungen zur Meldeverpflichtung von schwerwiegenden Transfusionsreaktionen ( $\$ 16$ Abs. 2 TFG, §63c AMG). Die Qualität der vom Paul-Ehrlich-Institut (PEI) veröffentlichten Daten kann aber nur so gut sein, wie die Meldedisziplin es zulässt.

In Bezug auf TRALI weist der aktuelle „Hämovigilanz-Bericht des Paul-Ehrlich-Instituts“ [6] Erfreuliches aus: Sowohl bei den gemeldeten immunogenen TRALI-Reaktionen als auch den assoziierten Todesfällen kam es zu einem drastischen Rückgang. Dies ist nicht etwa als Ausdruck einer schlechteren Meldedisziplin, sondern als Folge einer vom PEI seit September 2009 verbindlich angeordneten Spenderselektion für GFP zu sehen.

Spätestens nach der aufmerksamen Lektüre der Übersichtsarbeit von Tank et al. [7] in dieser Ausgabe von Der Anaesthesist werden Sinn, Zweck und Wirksamkeit dieser Anordnung der Bundes- oberbehörde klar. Besonders lesenswert ist dieser Beitrag aber auch deshalb, weil er mit sehr viel klinischem Bezug eine oft unterschätzte spezifische Transfusionsreaktion umfassend und nach dem aktuellen Stand der Wissenschaft beschreibt. Dazu gehört auch die Erkenntnis, dass zwar mit der Spenderselektion bei der Gewinnung von GFP eine sehr wirksame Risikoreduktion gelungen ist, TRALI aber auch durch die Transfusion von Thrombozyten- und Erythrozytenkonzentraten ausgelöst werden kann.

Seien wir also auch weiterhin „hämovigilant“!

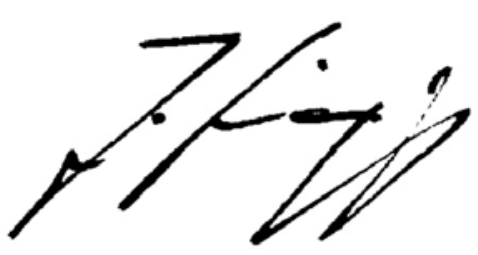

J. Biscoping

Federführender des Arbeitsausschusses Bluttransfusion der Deutschen Gesellschaft für Anästhesiologie und Intensivmedizin e. V. (DGAI) und des Berufsverbands Deutscher Anästhesisten e. V. (BDA)

\section{Korrespondenzadresse}

Prof. Dr. J. Biscoping

Klinik für Anaesthesie und Operative Intensivmedizin, St. Vincentius-Kliniken gAG Steinhäuserstr. 18, 76135 Karlsruhe j.biscoping@vincentius-ka.de

Interessenkonflikt. Der korrespondierende Autor gibt an, dass kein Interessenkonflikt besteht. 


\section{Literatur}

1. Bux J (2005) Transfusion-related acute lung injury (TRALI): a serious adverse event of blood transfusion. Vox Sang 89:1-10

2. Popovsky MA, Abel MD, Moore SB (1983) Transfusion-related acute lung injury associated with passive transfer of antileukocyte antibodies. Am Rev Respir Dis 128:185-189

3. Sachs UJ (2007) Pathophysiology of TRALI: current concepts. Intensive Care Med 33 (Suppl 1):3-11

4. Sachs UJ, Kauschat D, Bein G (2005) White blood cell-reactive antibodies are undetectable in solvent/detergent plasma. Transfusion 45:1628-1631

5. Working Expert Group (WEG) \& Writing Group, on behalf of the SHOT Steering Group (2011) Annual report 2010. http://www.shotuk.org/home.htm

6. Funk M, Günay S (2012) Haemovigilance Report of the Paul-Ehrlich-Institut 2010: Assessment of the Reports of Serious Adverse Transfusion Reactions pursuant to Section 63 c AMG (Arzneimittelgesetz, German Medicinal Products Act). Paul-Ehrlich-Institut, Langen

7. Tank S, Sputtek A, Kiefmann R (2013) Transfusionsassoziierte akute Lungeninsuffizienz. Anaesthesist 62:254-260

\section{DGAI-Forschungsstipendium der Fresenius-Stiftung 2013}
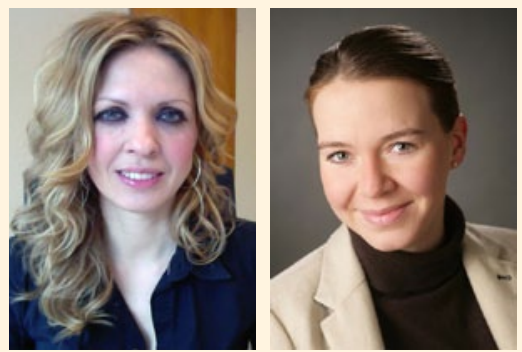

$\triangle$ Frau Dr. med. Val- $\triangle$ Frau Dr. med. Nanabona Mirakaj Maria Wagner

Das mit 20.000,- EUR dotierte DGAI-Forschungsstipendium der Fresenius-Stiftung wurde zu gleichen Teilen an Frau Dr. med. Valbona Mirakaj von der Klinik für Anästhesiologie und Intensivmedizin der Universität Tübingen und Frau Dr. med. Nana-Maria Wagner von der Klinik und Poliklinik für Anästhesiologie und Intensivtherapie Universitätsmedizin Rostock verliehen. Dr. Mirakaj wies mit ihrer Studie „Die Entzündungsresolution wird entscheidend durch Netrin-1 beeinflusst" grundlegende Mechanismen zur akuten Entzündungsreaktion nach, die zu neuartigen Behandlungswegen im intensivmedizinischen Bereich führen können.

Dr. Wagner beschreibt in ihrer Arbeit „Die pro-angiogene Wirkung einer TLR2 Inhibition geht mit einer verbesserten regenerativen Funktion knochenmarkstämmiger Stammzellen einher" einen neu entdeckten molekularen Mechanismus an der Schnittstelle von Inflammation und Angiogenese, der Ausgangspunkt zur Entwicklung therapeutischer Verbesserung der Durchblutung bei inflammatorischen Prozessen sein kann.

Die Arbeiten wurden auf den Wissenschaftlichen Arbeitstagen der Deutschen Gesellschaft für Anästhesiologie und Intensivmedizin vorgestellt. Die Preisverleihung fand auf dem Anästhesiekongress in Nürnberg statt.

Dr. Manfred Specker, Fresenius-Stiftung Bad Homburg

\section{Rudolf-Frey-Preis für Notfall- medizin 2013}

PD Dr. med. Jan-Thorsten Gräsner, Ärztlicher Leiter Notfallmedizin an der Klinik für Anästhesiologie und Operative Intensivmedizin am Universitätsklinikum Schleswig-Holstein, Campus Kiel erhält den diesjährigen RudolfFrey-Preis für Notfallmedizin. Der von der Deutschen Gesellschaft für Anästhesiologie und Intensivmedizin (DGAl) ausgelobte Forschungspreis wird für herausragende wissenschaftliche Leitungen auf dem Gebiet der Notfallmedizin verliehen. Gräsner erhält den Preis für seine Habilitationsarbeit zum „Aufbau und Analyseoption eines Reanimationsregisters als Instrument zur Optimierung präklinischer Reanimationsmaßnahmen".

Das Deutsche Reanimationsregister stellt aktuell die bundesweit größte Datenbank zur Erfassung, Analyse und dem Vergleich von Reanimationsmaßnahmen dar. Gräsner konnte mit seinen Arbeiten zeigen, dass Registerarbeiten klassische randomisierte klinische Studien sinnvoll ergänzen. Unter anderem entwickelte er mit seiner Arbeitsgruppe einen Prognosescore, der am Deutschen Reanimationsregister beteiligten Rettungsdiensten eine risikoadjustierte Beurteilung der eigenen Reanimationsleistungen ermöglicht. Die aus den Arbeiten abgeleiteten Erkenntnisse wurden direkt den Teilnehmern des Deutschen Reanimationsregisters in Form von automatisierten Auswerteoptionen zur Verfügung gestellt.

Quelle: Deutschen Gesellschaft für Anästhesiologie und Intensivmedizin (DGAl), www. dgai.de 